FACULDADE DE CIÊNCIAS ECONÔMICAS DA UFRGS Análise
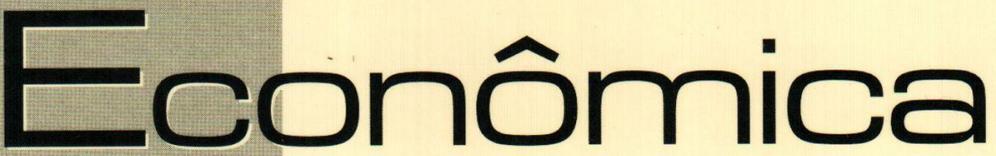

FLUTUACÃO CAMBIAL E METAS INFLACIONÁRIAS EM

ECONOMIAS EMERGENTES

OTAVIANO CANUTO E MárCIO HOLLAND

ATEORIA DE FORMACĆOO DE PRECOS E A TEORIA DOS PREÇOS DE

PRODUCAO

LOIZALBERTO ESTEVES

AECONOMIA EVOLUCIONISTA: UM CAPITULOSISTEMMICO DA

TEORIA ECONOMICA?

HUGOE. A. DA GAMACERQUEIRA

RELAÇ̃O INFLAÇÃO-PRODUTO NO BRASIL NO PERIODO PÓS-

REAL

MARCOSC. HOLANDA

DIVIIDA PÚBLICAMOBILLÍRIA FEDERAL BRASILEIRA: HISTÓRIA RECENTE E PERSPECTIVAS EXPLOSIVAS

ROGÉRIO MEURER E ROBERT WAYNE SAMOHYI

A CEPAL EA INTEGRACÃO REGIONAL LATINO-AMERICANA JACQUELINE A. HERNANDEZ HAFFNER

POPPER, HAYEK EA (IM)POSSIBILIDADE DE PREDIÇŌES ESPECIFICAS EM CIEENCIAS SOCIAIS

BRENA PAULA MAGNO FERNANDEZ

DINÂMICA RECENTE DO PROCESSO DE INCUBACG̃O DE

EMPRESAS DE BASE TECNOLOGICA NO BRASIL

EDUARDO GONÇALVES

TRIBUTACĀO COM SACRIF́CIO EQUUITATIVO: O CASO DO IMPOSTO DE RENDA PESSOA FISICA

STEFANO FLORISSI E EDUARDO PONTUAL RIBEIRO

A NOVATEORIA DO IMPÉRIOE AS VELHAS TEORIAS DO

IMPERIALISMO

GENTIL CORAZZA

Ô ABREALAS - A NOVA INSERÇÃO DO BRASIL NA ECONOMIA MUNDIAL

EMLLLANO LUIS KLEN

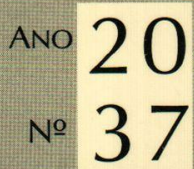

Março, 2002 
UNIVERSIDADE FEDERAL DO RIO GRANDE DO SUL

Reitora: Profa. Wrana Maria Panizzi

FACULDADE DE CIÊNCIAS ECONÔMICAS

Diretor: Prof. Pedro Cézar Dutra Fonseca

CENTRO DE. ESTUDOS E PESQUISAS ECONÔMICAS

Diretor: Prof. Gentil Corazza

DEPARTAMENTO DE CIÊNCIAS ECONÔMICAS

Chefe: Prof. Luiz Alberto Oliveira Ribeiro de Miranda

DAPARTAMENTO DE CIÊNCIAS CONTÁBEIS E ATUARIAIS

Chefe: João Marcos Leão da Rocha

CURSO DE PÓS-GRADUAÇÃO EM ECONOMIA

Coordenador: Prof. Eduardo Pontual Ribeiro

CURSO DE PÓS-GRADUAÇÃO EM ECONOMIA RURAL

Coordenador: Prof Jalcione Almeida

CONSELHO EDITORIAL: Carlos G. A. Mielitz Netto (UFRGS), Eduardo A. Maldonado Filho (UFRGS), Eduardo P. Ribeiro (UFRGS), Eleutério F. S. Prado (USP), Eugênio Lagemann (UFRGS), Fernando Cardim de Carvalho (UFRJ), Fernando Ferrari Filho (UFRGS), Fernando de Holanda Barbosa (FGV/RJ), Flávio Vasconcellos Comim (UFRGS), Gentil Corazza (UFRGS), Giácomo Balbinotto Neto (UFRGS), Gustavo Franco (PUC/RJ), Jan A. Kregel (Università di Bologna e John Hopkins University), João Rogério Sanson (UFSC), Joaquim Pinto de Andrade (UnB), Jorge Paulo Araújo (UFRGS), Juan H. Moldau (USP), Marcelo S. Portugal (UFRGS), Maria Alice L.ahorgue (UFRGS), Paul Davidson (University of Tennessee), Paulo Dabdab Waquil (UFRGS), Pedro Cézar Dutra Fonseca (UFRGS), Philip Arestis (South Bank University), Roberto C. Moraes (UFRGS), Ronald Otto Hillbrecht (UFRGS), Sabino da Silva Porto Jr. (UFRGS), Stefano Florissi (UFRGS), Werner Baer (Univ, of Illinois at Urbana-Champaign).

COMISSÃO EDITORIAL: Eduardo Augusto Maldonado Filho, Fernando Ferrari Filho, Gentil Corazza, Marcelo Savino Portugal, Paulo Dabdab Waquil; Roberto Camps Moraes.

EDITOR: Fernando Ferrari Filho

EDITOR ADJUNTO: Gentil Corrazza

SECRETÁRIA: Vanessa Hoffmann de Quadros

REVISÃO DE TEXTOS: Vanete Ricacheski

FUNDADOR: Prof. Antônio Carlos Santos Rosa

Os materiais publicados na revista Análise Econômica são da exclusiva responsabilidade dos autores. É permitida a reprodução total ou parcial dos trabalhos, desde que seja citada a fonte. Aceita-se permuta com revistas congêneres. Aceitam-se, também, livros para divulgação, elaboração de resenhas e recensões. Toda correspondência, material para publicação (vide normas na terceira capa), assinaturas e permutas devem ser dirigidos ao seguinte destinatário:

PROF FERNANDO FERRARI FILHO

Revista Análise Econômica - Av. João Pessoa, 52 CEP 90040-000 PORTO ALEGRE - RS, BRASIL Telefones: (051) 316-3348 e 316-3440 - Fax: (051) 316-3990

E-mail: rae@vortex ufrgs br

Análise Econômica

Ano 20, n 37 , março, 2002 - Porto Alegre

Faculdade de Ciências Econômicas, UFRGS, 2000

Periodicidade semestral, março e setembro.

1. Teoria Econômica - Desenvolvimento Regional Economia Agrícola - Pesquisa Teórica e Aplicada -

Periódicos. I. Brasil.

Faculdade de Ciências Econômicas,

Universidade Federal do Rio Grande do Sul.

CDD 330.05

CDU 33 (81) (05) 


\section{Ô Abre Alas - A nova inserção do Brasil na Economia Mundial}

Reinaldo Gonçalves, em "Ô Abre Alas", faz uma análise crítica das transformações globais, principalmente nas duas últimas décadas, e disserta sobre um novo padrão de inserção internacional da economia brasileira na economia mundial. Mais do que uma discussão sobre o "drama hamletiano de abrir ou fechar a economia", o autor apresenta diretrizes para uma inserção econômica soberana do Brasil neste contexto.

Com idéias e premissas opostas ao pensamento neoliberal e divergente também daquilo que chama de "sabedoria convencional", o autor inicia sua obra, dividida em seis capítulos, fazendo uma breve introdução a respeito de mudanças estruturais e novos paradigmas nos planos político, ideológico, militar-estratégico, tecnológico, organizacional e institucional. Nos quatro primeiros capítulos, ele analisa transformações ocorridas nas áreas comercial, monetária, e financeira e produtiva além de temas atuais como, internacionalização da produção e globalização, reação estratégica de empresas transnacionais, integração econômica, regionalismo e multilateralismo, Rodada Uruguai do GATT, globalização financeira, endividamento externo e reforma do sistema de Bretton Woods.

Nos dois capítulos finais, Gonçalves trata da inserção internacional do Brasil especificamente, discutindo introdutoriamente os reflexos das mudanças e dos temas citados acima na economia brasileira. $\mathrm{O}$ economista fecha a obra dissertando sobre as perspectivas e princípios básicos para inserção internacional brasileira na economia mundial. $\mathrm{O}$ autor, retoma um conceito que anda esquecido: o interesse nacional. Para Gonçalves, o Brasil é uma das economias mais internacionalizadas do mundo e com grande vulnerabilidade externa. Sendo assim, a inserção internacional não deve ser vista como um fim em si mesma, mas na sua funcionalidade dentro de um projeto de desenvolvimento nacional, projeto este que deve sobrepor os interesses da maioria da

\footnotetext{
- Aluno da Graduação do curso de Ciências Econômicas da Universidade Federal do Rio Grande do Sul.
} 
população, e subordinar o interesse privado ao público, onde o Estado é o principal ator das relações econômicas internacionais, pois cabe a ele a função de formular e executar um projeto nacional. Estas são algumas das premissas do autor para nossa inserção econômica.

Neste sentido, Reinaldo Gonçalves encerra sua obra dizendo que seu objetivo não é formular um "modelo completo", mas sim analisar dados e discutir perspectivas, trazendo assuntos que estão fora do debate e que podem ser fundamentais para a formulação de um projeto nacional. Fica implícito durante todo o trabalho que o autor tem profundo interesse em polemizar e fazer com que se discuta muito mais além da idéia de "abrir ou fechar a economia". Para todos os interessados em um conhecimento maior sobre o sistema econômico mundial, e assim como novas idéias sobre a inserção internacional do Brasil, "Ô Abre Alas" é uma ótima leitura.

GONÇALVES, Reinaldo. Ô Abre Alas - A Nova Inserção do Brasil na Economia Mundial $1^{\text {a }}$ edição em 1994, Editora Relume Dumará. 